Discrete Comput Geom 34:697-706 (2005)

DOI: $10.1007 / \mathrm{s} 00454-005-1188-9$

\title{
Acute Triangulations of Polygons
}

\author{
Liping Yuan \\ College of Mathematics and Information Science, Hebei Normal University, \\ 050016 Shijiazhuang, People's Republic of China \\ lpyuan88@yahoo.com, lpyuan@hebtu.edu.cn \\ and \\ Department of Mathematics, University of Dortmund, \\ 44221 Dortmund, Germany
}

\begin{abstract}
In this paper we present upper bounds for the number of triangles in a triangulation of an $n$-gon, imposing the condition that all triangles used are non-obtuse, or just acute. This is based on work by Bern et al. [2] and Maehara [8], and strengthens their results.
\end{abstract}

\section{Introduction}

By a triangulation of a (planar) polygon, we mean a finite set of triangles covering the polygon in such a way that any two distinct triangles are either disjoint or intersect in a single common vertex or edge. An acute (non-obtuse) triangulation of a polygon is a triangulation whose triangles have all their angles less (not larger) than $\pi / 2$. Burago and Zalgaller [3] proved that every polygon admits an acute triangulation. Moreover, Bern et al. [2] gave an algorithm for triangulating $n$-gons into $O(n)$ non-obtuse triangles. Burago and Zalgaller [3] and, independently, Manheimer [9] proved that every obtuse triangle can be triangulated into seven acute triangles, and seven is the minimum number. Cassidy and Lord [4] showed that every square can be triangulated into eight acute triangles, and eight is the minimum number. Hangan et al. [6] extended this result to any rectangle. Maehara [7] proved that every quadrilateral can be triangulated into ten acute triangles, and ten is best possible. By the result of Burago and Zalgaller, every polygon admits an acute triangulation. However, their proof cannot be used to obtain a good estimate of the actual number of triangles in it. Maehara [8] proved the existence of an acute triangulation for any polygon on the basis of the existence of a non-obtuse triangulation. At the same time, he gave the upper bound $2 \cdot 6^{5} \mathrm{~N}$ for the number of triangles in the acute triangulation, where $N$ denotes the number of triangles in the existing non-obtuse triangulation. 


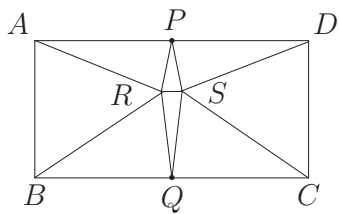

(a)

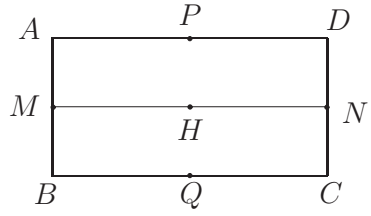

(b)

Fig. 1. The acute triangulations of rectangles.

In Section 2 we present some preparatory propositions. In Section 3 we describe another way to acutely triangulate a polygon based on the existence of a non-obtuse triangulation. We obtain the upper bound $24 \mathrm{~N}$ for the number of triangles in the acute triangulation. In Section 4 we find a concrete upper bound for the size of a non-obtuse triangulation of an $n$-gon, obtaining $N \leq 106 n-216$ on the basis of the method used in [2]. This implies that every $n$-gon can be triangulated into at most $24 \cdot(106 n-216)$ acute triangles.

\section{Preliminaries}

Hangan et al. [6] considered acute triangulations of the rectangles.

Proposition 1 [6]. Every rectangle can be triangulated into eight acute triangles.

The construction in [6] (see Fig. 1(a)) immediately implies the following result (see Fig. 1(b)).

Proposition 2. Every rectangle can be triangulated into 16 acute triangles such that there is precisely one new vertex introduced on each side, and the new vertex is exactly the midpoint of the side.

For the sake of convenience, we call this acute triangulation (described in Proposition 2) a basic triangulation of the rectangle.

Naturally, if a triangulation $\mathcal{T}$ is acute, then any perturbation of $\mathcal{T}$ which is small enough leaves $\mathcal{T}$ acute.

Let $T=A B C$ be a triangle and let $M, N, P$ be the midpoints of $A B, B C, C A$, respectively. Then $M N, N P, M P$ divide $A B C$ into four congruent triangles which are similar to $T$. We call such a triangulation an elementary subdivision of $T$.

\section{Acute Triangulations of Polygons}

In a triangulation, two triangles sharing a common edge are called adjacent. Let $\Gamma$ be a polygon and let $\mathcal{T}$ be a non-obtuse triangulation of $\Gamma$. We now describe a new way of transforming $\mathcal{T}$ into an acute triangulation of $\Gamma$. 

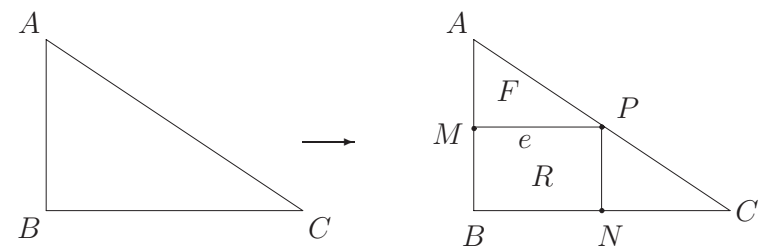

Fig. 2. The division of a right triangle.

Step 1. Subdivide $\mathcal{T}$ to $\mathcal{T}_{1}$.

Rule 1.1. Any acute triangle in $\mathcal{T}$ will be divided into four acute triangles by an elementary subdivision.

Rule 1.2. Any right triangle in $\mathcal{T}$ will be divided into a rectangle and two right triangles as shown in Fig. 2, where the point on each side is its midpoint.

Then we obtain a face-to-face tiling $\mathcal{T}_{1}$ of $\Gamma$ which consists of acute triangles, rectangles and right triangles. Let $\mathcal{S}_{1}, \mathcal{R}_{1}$ and $\mathcal{F}_{1}$ denote the family of all acute triangles, all rectangles and all right triangles in $\mathcal{T}_{1}$. Then $\mathcal{T}_{1}=\mathcal{S}_{1} \cup \mathcal{R}_{1} \cup \mathcal{F}_{1}$. Each triangle $F \in \mathcal{F}_{1}$ is obtained according to Rule 1.2 (see Fig. 2) and has a rectangle $R$ as a neighbor. This rectangle $R$ is called the basic rectangle of $F$ and the common edge of $F$ and $R$ is called the basic edge of $F$. Thus any $F \in \mathcal{F}_{1}$ has one and only one basic rectangle and basic edge.

Step 2. Subdivide $\mathcal{T}_{1}$ into $\mathcal{T}_{2}$.

Rule 2.1. Apply an elementary subdivision to every element in $\mathcal{S}_{1} \cup \mathcal{F}_{1}$.

Rule 2.2. Apply a basic triangulation to every rectangle in $\mathcal{R}_{1}$.

Then we obtain a non-obtuse triangulation $\mathcal{T}_{2}$ of $\Gamma$. Next we prove that $\mathcal{T}_{2}$ can be converted into an acute triangulation of $\Gamma$.

Let $\mathcal{S}_{2}, \mathcal{R}_{2}, \mathcal{F}_{2}$ denote all the triangles in $\mathcal{T}_{2}$ which are obtained from $\mathcal{S}_{1}, \mathcal{R}_{1}, \mathcal{F}_{1}$, respectively. Every triangle in $\mathcal{S}_{2} \cup \mathcal{R}_{2}$ is acute. Hence we only need to consider the triangles in $\mathcal{F}_{2}$. Notice that the right triangles in $\mathcal{F}_{2}$ are obtained from $\mathcal{F}_{1}$ by elementary subdivision, so we can classify them according to their corresponding right triangles in $\mathcal{F}_{1}$.

For any acute triangles $S \in \mathcal{S}_{1}$, let $\varphi(S)$ denote the family of four acute triangles obtained from the elementary subdivision of $S$. For any rectangle $R \in \mathcal{R}_{1}$, let $\varphi(R)$ denote the family of 16 acute triangles obtained from the basic triangulation of $R$. For any right triangles $F \in \mathcal{F}_{1}$, let $\varphi(F)$ denote the family of four right triangles obtained from the elementary subdivision of $F$. We call the three triangles in $\varphi(F)$ which have non-empty intersection with the basic edge $e$ of $F$ the adjacent triangles with respect to $e$, and the other one which is disjoint from $e$ the opposite triangle with respect to $e$. Moreover, if $\mathcal{S}_{1}=\left\{S_{1}, S_{2}, \ldots, S_{t}\right\}, \mathcal{F}_{1}=\left\{F_{1}, F_{2}, \ldots, F_{n}\right\}$ and $\mathcal{R}_{1}=\left\{R_{1}, R_{2}, \ldots, R_{m}\right\}$, 


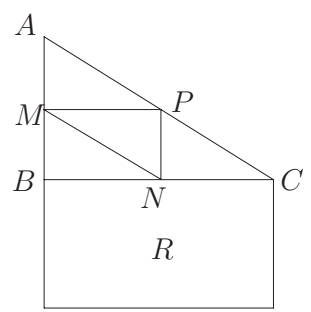

Fig. 3. The basic sliding of $\varphi(F)$.

then $\mathcal{S}_{2}=\varphi\left(S_{1}\right) \cup \varphi\left(S_{2}\right) \cup \cdots \cup \varphi\left(S_{t}\right), \mathcal{F}_{2}=\varphi\left(F_{1}\right) \cup \varphi\left(F_{2}\right) \cup \cdots \cup \varphi\left(F_{n}\right)$ and $\mathcal{R}_{2}=\varphi\left(R_{1}\right) \cup \varphi\left(R_{2}\right) \cup \cdots \cup \varphi\left(R_{m}\right)$.

Lemma 1. For any $F \in \mathcal{F}_{1}$ with basic rectangle $R \in \mathcal{R}_{1}$ and basic edge $e$, the triangulation can be perturbed so that all triangles in $\varphi(F) \cup \varphi(R)$ except the opposite triangle with respect to $e($ in $\varphi(F))$ become or remain acute.

Proof. Let $F=A B C$ with basic edge $B C$, and let $N$ be the midpoint of $B C$, as shown in Fig. 3. We can slide $N$ slightly in direction $\overrightarrow{N B}$ such that both $M P N$ and $P N C$ become acute triangles while all triangles in $\varphi(R)$ remain acute. Then we slide $N$ slightly in direction $\overrightarrow{B A}$ such that $M B N$ becomes acute while $M P N, P N C$ and all triangles in $\varphi(R)$ remain acute.

For each $F \in \mathcal{F}_{1}$, we call the sliding described in the proof of Lemma 1 the basic sliding of $\varphi(F)$.

Let $\mathcal{F}_{1}=\left\{F_{1}, F_{2}, \ldots, F_{n}\right\}$, and let $e_{i}$ denote the basic edge of $F_{i}(i=1,2, \ldots, n)$. Next we convert the triangulation $\mathcal{T}_{2}$ to $\mathcal{T}_{3}$ by the following step.

Step 3. Apply the basic sliding to each $\varphi\left(F_{i}\right)$, where $i=1,2, \ldots, n$.

After Step 3, we know that in each $\varphi\left(F_{i}\right)(i=1,2, \ldots, n)$, only the opposite triangle with respect to $e_{i}$ is not changed into an acute triangle. We denote by $\varphi_{3}\left(F_{i}\right)$ the set of four triangles in $\mathcal{T}_{3}$ resulting from $\varphi\left(F_{i}\right)$. Then there are exactly $n$ right triangles in $\mathcal{T}_{3}$, one in each of $\varphi_{3}\left(F_{i}\right)(i=1,2, \ldots, n)$.

If $F \in \mathcal{F}_{1}$ has no adjacent right triangle, then we call it an isolated triangle. Let $F_{i}$ and $F_{j}$ be two adjacent triangles in $\mathcal{F}_{1}$, if they have a common leg (hypotenuse), we call them of Type I (or of Type II); otherwise, we call them of Type III, see Fig. 4.

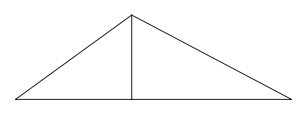

(a) Type I

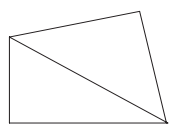

(b) Type II

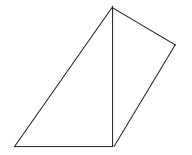

(c) Type III

Fig. 4. Three kinds of adjacent triangles in $\mathcal{F}_{1}$. 


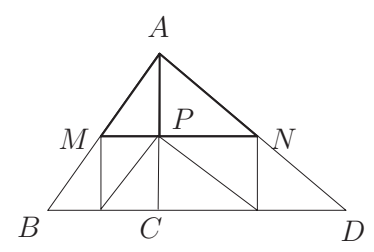

Fig. 5. $\quad F_{i}$ and $F_{j}$ are of Type I.

Lemma 2. For any two adjacent triangles $F_{i}$ and $F_{j}$ in $\mathcal{F}_{1}$, we can transform all the triangles in $\varphi_{3}\left(F_{i}\right) \cup \varphi_{3}\left(F_{j}\right)$ into acute triangles, leaving unchanged all other triangles in $\mathcal{T}_{3}-\left(\varphi_{3}\left(F_{i}\right) \cup \varphi_{3}\left(F_{j}\right)\right)$.

Proof. There are three cases to consider.

Case 1: $F_{i}$ and $F_{j}$ are of Type $I$. From the given condition we know that $e_{i}$ and $e_{j}$ must be collinear. Suppose that $F_{i}=A B C$ and $F_{j}=A C D$, as shown in Fig. 5. Then $e_{i}=B C$, $e_{j}=C D$. Thus $A M P$ and $A N P$ are the two remaining right triangles in $\varphi_{3}\left(F_{i}\right) \cup \varphi_{3}\left(F_{j}\right)$. Now we can slide $P$ slightly in direction $\overrightarrow{A C}$ such that both $A M P$ and $A N P$ become acute while all other triangles in $\varphi_{3}\left(F_{i}\right) \cup \varphi_{3}\left(F_{j}\right)$ remain acute.

Case 2: $F_{i}$ and $F_{j}$ are of Type II. Without loss of generality, we may assume that $F_{i}=A B C$ with basic edge $B C$ and $F_{j}=A C D$. Then $e_{j}=C D$. Hence $A M O$ and $A N O$ are the two remaining right triangles in $\varphi_{3}\left(F_{i}\right) \cup \varphi_{3}\left(F_{j}\right)$, as shown in Fig. 6. Now we can slide $O$ slightly in direction $\overrightarrow{C A}$ such that all the triangles in $\varphi_{3}\left(F_{i}\right) \cup \varphi_{3}\left(F_{j}\right)$ are acute.

Case 3: $F_{i}$ and $F_{j}$ are of Type III. Without loss of generality, we may assume that the common edge is a leg of $F_{i}$ and the hypotenuse of $F_{j}$.

Let $F_{i}=A B C$ and $F_{j}=A C D$, then $e_{i}=B C, e_{j}=C D$. Thus $A M O$ and $A N O$ are the two remaining right triangles in $\varphi_{3}\left(F_{i}\right) \cup \varphi_{3}\left(F_{j}\right)$, as shown in Fig. 7. We establish an $x-y$ coordinate system with $O$ as the origin, $M O$ as the $x$-axis and $C A$ as the $y$-axis (also see Fig. 7). Let $k_{O N}$ denote the slope of the line $O N$. Then $k_{O N} \neq 0$. Let $\mathbf{O}$ be the circumscribed circle of $A M O$ and let $l$ be the tangent line of $\mathbf{O}$ at point $O$, then the slope $k_{l}$ of $l$ is not zero.

If $k_{O N} \leq k_{l}$, we can slide $O$ slightly in direction $\overrightarrow{O N}$ at first such that $A M O$ becomes acute while $A N O$ remains right and the rest of triangles in $\varphi_{3}\left(F_{i}\right) \cup \varphi_{3}\left(F_{j}\right)$ remain acute. Then we slide $O$ slightly in direction $\overrightarrow{D A}$ such that all the triangles in $\varphi_{3}\left(F_{i}\right) \cup \varphi_{3}\left(F_{j}\right)$ are acute.

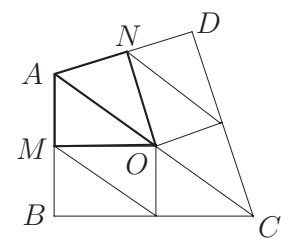

Fig. 6. $\quad F_{i}$ and $F_{j}$ are of Type II. 


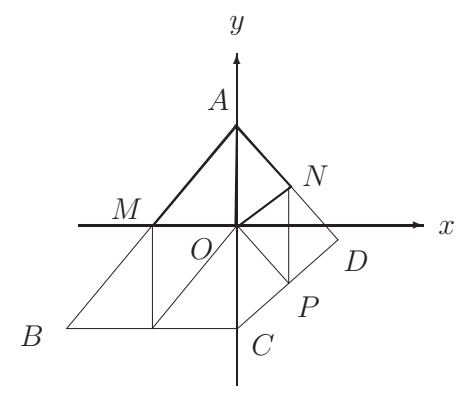

Fig. 7. $\quad F_{i}$ and $F_{j}$ are of Type III.

If $k_{O N}>k_{l}$, similarly we can slide $O$ slightly in direction $\overrightarrow{N O}$ at first and then slide $O$ slightly in direction $\overrightarrow{D A}$ such that all the triangles in $\varphi_{3}\left(F_{i}\right) \cup \varphi_{3}\left(F_{j}\right)$ are acute.

Finally, it is easy to check that all the slidings mentioned above leave all other triangles in $\mathcal{T}_{3}-\left(\varphi_{3}\left(F_{i}\right) \cup \varphi_{3}\left(F_{j}\right)\right)$ unchanged. The proof is complete.

For any two adjacent triangles $F_{i}$ and $F_{j}$ in $\mathcal{F}_{1}$, we call the sliding described in the proof of Lemma 2 the basic pair sliding of $F_{i}$ and $F_{j}$.

Step 4. Apply the basic pair sliding in $\mathcal{T}_{3}$ as follows, and denote the obtained triangulation by $\mathcal{T}_{4}$ :

Step 4.1. Apply the basic pair sliding to all Type I adjacent triangles in $\mathcal{F}_{1}$, and denote all the triangles involved in this step by $\mathcal{P}_{1}$.

Step 4.2. Apply the basic pair sliding to all Type II adjacent triangles in $\mathcal{F}_{1}-\mathcal{P}_{1}$, and denote all the triangles involved in this step by $\mathcal{P}_{2}$.

Step 4.3. If there are two triangles in $\mathcal{F}_{1}-\left(\mathcal{P}_{1} \cup \mathcal{P}_{2}\right)$ which are adjacent triangles of Type III, then we apply the basic pair sliding to them. Repeat this kind of work until there are no Type III adjacent triangles. Denote all the triangles involved in this step by $\mathcal{P}_{3}$.

Let $\mathcal{P}_{0}=\mathcal{F}_{1}-\left(\mathcal{P}_{1} \cup \mathcal{P}_{2} \cup \mathcal{P}_{3}\right)$. If $\mathcal{P}_{0}=\emptyset$, then $\mathcal{T}_{4}$ is an acute triangulation of $\Gamma$. If $\mathcal{P}_{0} \neq \emptyset$, then any $F \in \mathcal{P}_{0}$ is either an isolated triangle in $\mathcal{F}_{1}$ or its adjacent triangle (of Type III) admits an acute triangulation. Let $F=A B C$ be a triangle in $\mathcal{P}_{0}$ with basic edge $B C$. If there is an $X \in \mathcal{T}_{1}$ which has the common side $A B$ with $F$, then all the triangles in $\varphi(X)$ are acute. As a result, we can slide $N$ slightly in direction $\overrightarrow{A B}$ such that all the triangles in $\varphi_{3}(F) \cup \varphi_{3}(X)$ become acute (see Fig. 8). If there is not such an $X$, then $A B$ lies on a side of $\Gamma$ and we can perform a similar sliding. Applying this kind of sliding to each element of $\mathcal{P}_{0}$, we transform $\mathcal{T}_{4}$ into an acute triangulation of $\Gamma$.

Combining the above discussion, we immediately have the following theorem.

Theorem 1. Every polygon admits an acute triangulation.

Furthermore, considering the number of triangles in the obtained acute triangulation, we have the following result. 


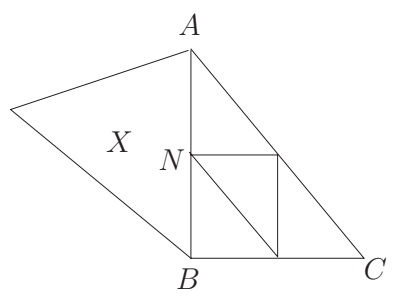

Fig. 8. A triangle in $\mathcal{P}_{0}$.

Theorem 2. If a polygon can be triangulated into $N$ non-obtuse triangles, then it can be triangulated into at most $24 \mathrm{~N}$ acute triangles.

Proof. Let $\mathcal{T}$ be a non-obtuse triangulation of a polygon and let $v_{1}\left(v_{2}\right)$ denote the number of all acute (right) triangles in $\mathcal{T}$, then $N=v_{1}+v_{2}$. Notice that $\left|\mathcal{S}_{1}\right|=4 v_{1}$, $\left|\mathcal{R}_{1}\right|=v_{2}$ and $\left|\mathcal{F}_{1}\right|=2 v_{2}$ in $\mathcal{T}_{1}$; we have $\left|\mathcal{T}_{2}\right|=4\left(\left|\mathcal{S}_{1}\right|+\left|\mathcal{F}_{1}\right|\right)+16\left|\mathcal{R}_{1}\right|=16 v_{1}+24 v_{2}=$ $16\left(v_{1}+v_{2}\right)+8 v_{2} \leq 24 N$. This implies that $\left|\mathcal{T}_{4}\right|=\left|\mathcal{T}_{3}\right|=\left|\mathcal{T}_{2}\right| \leq 24 N$. The proof is complete.

\section{Non-Obtuse Triangulations of Polygons}

In [2] it was proved that every $n$-gon can be triangulated into $O(n)$ acute triangles. In this section we obtain a concrete upper bound for the size of a non-obtuse triangulation of an $n$-gon based on work in [2].

Let $\Gamma$ be an $n$-gon (without a hole) with $r$ concave corners. We start with the basic method, namely, the disk packing presented in [2].

Firstly, we pack the disks at corners. At every convex vertex of $\Gamma$, we add a small disk tangent to both edges, as shown in Fig. 9(a). At every concave corner of $\Gamma$, we add two disks of equal radii, tangent to the edges and tangent to the angle bisector at the corner, as shown in Fig. 9(b). We choose radii small enough such that the disks lie within $\Gamma$, and any two disks from two distinct vertices are disjoint. This step isolates a small three- or four-sided remainder region at each corner of $\Gamma$. The large remainder region is a simply connected arc-gon with $2 n+r$ sides, where an arc-gon is a simple polygon with arcs of circles as sides, and the circles may have various radii, including infinite ones (which correspond to straight sides).

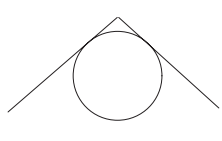

(a)

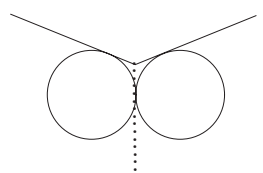

(b)

Fig. 9. Adding disks at corners. 


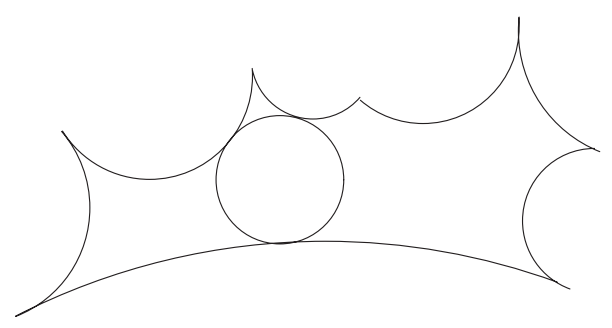

Fig. 10. A disk tangent to three edges of an arc-gon.

Let $A$ be a simply connected $m$-vertex arc-gon. To subdivide $A$, we add a disk tangent to three sides, not all of which are consecutive (it is possible that it is tangent to more than three sides), as shown in Fig. 10. Then we have the following result.

Lemma 3 [2]. It is possible to reduce the numbers of sides of each of the remainder regions to at most four, by packing at most $m-4$ non-overlapping disks into the arc-gon $A$.

At this point, the polygonal region $\Gamma$ has been partitioned into disks and remainder regions with three or four sides, either straight or circular arcs. It is clear that there are three kinds of such regions, namely, remainder regions with vertices of $\Gamma$ (denoted by $R_{P_{i}}$, where $P_{i}$ is a vertex of $\left.\Gamma(i=1,2, \ldots, n)\right)$, remainder regions with three sides (denoted by $R_{3}$ ) and remainder regions with four sides (denoted by $R_{4}$ ). Moreover, each circular arc of a remainder region $R$ is naturally associated with a pie-shaped sector. We denote the union of $R$ and its associated sectors by $R^{+}$. These augmented remainder regions define a decomposition of $\Gamma$ into simple polygons with disjoint interiors, as shown in Fig. 11.

Now we present several results in [2] concerning the triangulation of each $R^{+}$obtained. New vertices introduced to triangulate $\Gamma$ are called additional points (Steiner points in the terminology of [2]). We call an additional point in an augmented region $R^{+}$safe if it lies either in the interior to $R^{+}$or on the boundary of $\Gamma$.

Lemma 4 [2]. Let $P_{i}(i=1,2, \ldots, n)$ be a vertex of an $n$-gon $\Gamma$. If $P_{i}$ is a convex (resp. concave) corner, then $R_{P_{i}}^{+}$can be triangulated into two (resp. four) right triangles such that there is no new vertex introduced in the triangulation.

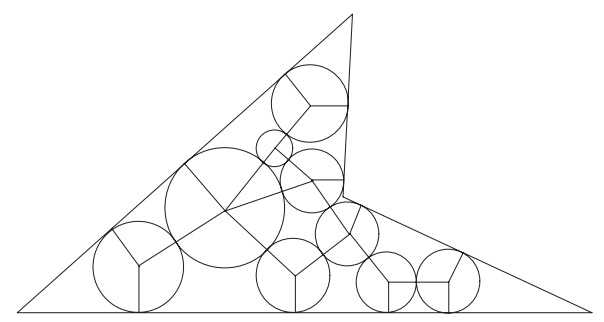

Fig. 11. Decomposition of $\Gamma$ into simple polygons with disjoint interiors. 
Lemma 5 [2]. Every $R_{3}^{+}$(resp. $R_{4}^{+}$) can be triangulated into at most six (resp. 28) right triangles, adding only safe additional points.

Combining Lemma 3 to Lemma 5 we know that for every $m$-arc-gon $A, A^{+}$admits a non-obtuse triangulation (adding only safe additional points). Therefore $\Gamma$ admits a non-obtuse triangulation. Let $\varphi(m)$ denote the number of triangles in a non-obtuse triangulation of $A^{+}$(where $A$ is an $m$-arc-gon), then Lemma 5 implies that $\varphi(3) \leq 6$ and $\varphi(4) \leq 28$. Moreover, we have

Lemma 6. For every $m \geq 4, \varphi(m) \leq 34 m-108$.

Proof. We prove this by induction on $m$. The base is $\varphi(4) \leq 28$. Now we suppose that the conclusion holds for all $4 \leq k<m$.

Let $A$ be an arc-gon with $m$ sides, and let $\mathbf{O}$ be the first disk packed in $A$. We assume that $\lambda(\lambda \geq 3)$ sides of $A$ are tangent to $\mathbf{O}$. Then $A$ is divided into one disk and $\lambda$ arc-gons having $k_{1}, k_{2}, \ldots, k_{\lambda}$ sides, respectively, with $3 \leq k_{1} \leq k_{2} \leq \cdots \leq k_{\lambda}$. Thus $\varphi(m)=\varphi\left(k_{1}\right)+\varphi\left(k_{2}\right)+\cdots+\varphi\left(k_{\lambda}\right)$. Since the disk divides $\lambda$ sides, and is itself divided in $\lambda$ places, we have $k_{1}+k_{2}+\cdots+k_{\lambda}=m+2 \lambda$.

Case 1: $\lambda=3$. Then by the rules of packing disks we know that not all of these three sides are consecutive in $A$ which implies that $3 \leq k_{1} \leq k_{2} \leq k_{3}<m$.

If $k_{1}=3$, then $\varphi(m)=\varphi(3)+\varphi\left(k_{2}\right)+\varphi\left(k_{3}\right) \leq 6+34 \times\left(k_{2}+k_{3}\right)-2 \times 108=$ $6+34 \times(m+3)-2 \times 108=34 m-108$.

If $k_{1} \geq 4$, then $\varphi(m)=\varphi\left(k_{1}\right)+\varphi\left(k_{2}\right)+\varphi\left(k_{3}\right) \leq 34 \times\left(k_{1}+k_{2}+k_{3}\right)-3 \times 108=$ $34 \times(m+6)-3 \times 108=34 m-120 \leq 34 m-108$.

Case 2: $\lambda \geq 4$. Then it is easy to see that $k_{\lambda}<m$. We may assume that among all of the $k_{i}(i=1, \ldots, \lambda)$, there are exactly $\lambda_{0}$ values which are equal to 3 , where $0 \leq \lambda_{0} \leq \lambda$. Then we have

$$
\begin{aligned}
\varphi(m) & =\varphi\left(k_{1}\right)+\varphi\left(k_{2}\right)+\cdots+\varphi\left(k_{\lambda}\right) \\
& \leq 6 \lambda_{0}+34 \cdot\left(k_{\lambda_{0}+1}+\cdots+k_{\lambda}\right)-108 \cdot\left(\lambda-\lambda_{0}\right) \\
& =6 \lambda_{0}+34 \cdot\left(m+2 \lambda-3 \lambda_{0}\right)-108 \cdot\left(\lambda-\lambda_{0}\right) \\
& =34 m-40 \lambda+12 \lambda_{0} \leq 34 m-28 \lambda \leq 34 m-28 \times 4 \\
& =34 m-112 \leq 34 m-108 .
\end{aligned}
$$

The proof is complete.

Theorem 3. Every n-gon admits a non-obtuse triangulation whose size is at most $106 n-216$.

Proof. Suppose that an $n$-gon $\Gamma$ admits a non-obtuse triangulation (obtained by the method described above) with size $N$. Let $r$ denote the number of concave corners of $\Gamma$. From the above discussion we know that $P=R_{P_{1}}^{+} \cup R_{P_{2}}^{+} \cup \cdots \cup R_{P_{n}}^{+} \cup R_{A}^{+}$, 
where $A$ is an arc-gon with $2 n+r$ sides. By Lemmas 4 and 6 we know that $N=$ $2(n-r)+4 r+\varphi(2 n+r) \leq 2 n+2 r+34(2 n+r)-108=70 n+36 r-108$. Noticing that $r \leq n-3$, we have $N \leq 106 n-216$.

The proof is complete.

Combining Theorems 2 and 3, we immediately obtain the following corollary.

Corollary 4. Every $n$-gon can be triangulated into at most $24 \cdot(106 n-216)$ acute triangles.

\section{Acknowledgement}

The author thanks Prof. Dr. Tudor Zamfirescu for his patient guidance.

\section{References}

1. B. S. Baker, E. Grosse and C. S. Rafferty, Nonobtuse triangulations of polygons, Discrete Comput. Geom., 3 (1988), 147-168.

2. M. Bern, S. Mitchell and J. Ruppert, Linear-size nonobtuse triangulations of polygons, Discrete Comput. Geom., 14 (1995), 411-428.

3. Y. D. Burago and V. A. Zalgaller, Polyhedral embedding of a net (in Russian), Vestnik Leningrad Univ., 15 (1960), 66-80.

4. C. Cassidy and G. Lord, A square acutely triangulated, J. Recreational Math., 13 (1980/81), 263-268.

5. M. Gardner, New Mathematical Diversions, Mathematical Association of America, Washington, DC, 1995.

6. T. Hangan, J. Itoh and T. Zamfirescu, Acute triangulations, Bull. Math. Soc. Sci. Math. Roumanie, 43(3-4) (91) (2000), 279-285.

7. H. Maehara, On acute triangulations of quadrilaterals, Proceedings of JCDCG 2000, Lecture Notes in Computer Science, Vol. 2098, Springer, Berlin, 2001, pages 237-243.

8. H. Maehara, Acute triangulations of polygons, European J. Combin., 23 (2002), 45-55.

9. W. Manheimer, Solution to Problem E1406: Dissecting an obtuse triangle into acute triangles, American Mathematical Monthly, 67 (1960), 923.

Received October 27, 2004, and in revised form May 5, 2005. Online publication August 4, 2005. 